\title{
Titration of submonolayer Au growth on $\mathrm{Si}(111)$
}

\author{
J. Kautz \\ Kamerlingh Onnes Laboratory, Leiden University, Niels Bohrweg 2, 2300 RA Leiden, The Netherlands \\ M. W. Copel and M. S. Gordon \\ IBM Research Division, Thomas J. Watson Research Center, Yorktown Heights, New York 10598, USA \\ R. M. Tromp \\ IBM Research Division, Thomas J. Watson Research Center, Yorktown Heights, New York 10598, USA \\ and Kamerlingh Onnes Laboratory, Leiden University, Niels Bohrweg 2, 2300 RA Leiden, The Netherlands \\ S. J. van der Molen* \\ Kamerlingh Onnes Laboratory, Leiden University, Niels Bohrweg 2, 2300 RA Leiden, The Netherlands
}

(Received 7 October 2013; published 14 January 2014)

\begin{abstract}
We study and analyze the growth of submonolayers of $\mathrm{Au}$ on $\mathrm{Si}(111)$ by a complementary set of surface techniques. Specifically, we focus on the $5 \times 2$ and the $\alpha \sqrt{3} \times \sqrt{3}$ structures. We determine the gold coverage of these structures as a function of temperature by means of low energy electron diffraction (LEED) and low energy electron microscopy (LEEM). These results are independently calibrated by ex-situ ion scattering experiments. This allows us to present a phase diagram for this system. Remarkably, for all temperatures considered $(820-1040 \mathrm{~K})$, we find a coverage for the $5 \times 2$ phase that is significantly $(\approx 10 \%)$ higher than the value of 0.6 monolayers which is assumed in the latest structural models. Therefore, a further refinement of the present picture of the quasi-one-dimensional $5 \times 2$ structure is required.
\end{abstract}

DOI: 10.1103/PhysRevB.89.035416

PACS number(s): 68.55.-a, 68.37.Nq, 68.43.Fg

\section{INTRODUCTION}

Submonolayers of gold on $\mathrm{Si}(111)$ have been studied with a wide range of surface analysis tools. ${ }^{1-18}$ Of particular interest is the $5 \times 2$ structure, which forms at low gold coverages. The highly anisotropic $5 \times 2$ structure consists of parallel rows that exhibit metallic behavior in one direction only. ${ }^{14,17,19}$ This makes it a possible model system to study the electronic properties of one-dimensional systems. The rows of this $5 \times 2$ structure can form in three directions, rotated by $120^{\circ}$ with respect to each other. ${ }^{20}$ Remarkably, the exact atomic arrangement of the $5 \times 2$ structure has been a long-standing problem in surface science. One of the main reasons for this is that the exact gold coverage of the $5 \times 2$ structure, i.e., the number of gold atoms per unit cell, has proven difficult to determine. Initially, the $5 \times 2$ structure was thought to exhibit a coverage of 0.4 monolayer (ML). ${ }^{4}$ Several models were subsequently proposed based on this value..$^{8,13,15,21}$ However, more advanced experimental studies pointed to a higher gold coverage, around 0.6 ML. ${ }^{16}$ This led Erwin et al. to improve their structural model in a 2009 contribution. ${ }^{17}$ After McAlinden et al. studied the influence of step edges, ${ }^{18}$ in 2013, yet another structural model was presented by Abukawa and Nishigaya. ${ }^{19}$ They performed reflection high-energy electron diffraction experiments and determined which structure was most consistent with the Patterson function derived from their data set. Still, their model was recently questioned in a paper by Hogan. ${ }^{22}$ All these groups constrain themselves to structures with a precise coverage of 0.6 ML of Au. However, in the experimental literature there is no consensus on this value. In fact, there is quite some discrepancy between the results from different groups, ranging from 0.57 to 0.67 ML. $4,16,18$ Clearly, to make a reliable structural model of the $5 \times 2$ surface structure, it is essential to obtain an accurate value for the gold coverage. We make use of the unique capabilities of low energy electron microscopy (LEEM), ${ }^{23}$ in combination with medium energy ion scattering(MEIS) and Rutherford back scattering (RBS), to reach that goal. Indeed, LEEM is perfectly suitable for our study, as it allows one to follow dynamic processes in real time, as well as to interrupt them at any moment. From our experiments, we find that the single-phase $5 \times 2$ structure occurs between coverage values of $0.65 \pm 0.02$ and $0.67 \pm 0.01$ monolayers, slightly depending on temperature. Since the coverage values we find for the $5 \times 2$ phase differ significantly from the one on which the two most recent models are based, the latter will need to be reconciled with our results and with each other.

In addition to studying the $5 \times 2$ structure, we investigated the $\alpha \sqrt{3} \times \sqrt{3}$ structure, which forms at higher gold coverages. $^{24,25}$ The latter structure coexists with the $5 \times 2$ structure for coverage values up to one monolayer. Upon heating, both the $5 \times 2$ and the $\alpha \sqrt{3} \times \sqrt{3}$ phases transform into a disordered $1 \times 1$ phase. Using four types of experiments, we map out a phase diagram for submonolayers of $\mathrm{Au}$ on $\mathrm{Si}(111)$.

\section{EXPERIMENTAL}

We combine LEEM with in-situ gold evaporation on $\mathrm{Si}(111)$ to quantitatively follow the growth of the $5 \times 2$ and the $\alpha \sqrt{3} \times \sqrt{3}$ structures in real time at a temperature of choice. By closing (opening) the Au evaporator shutter we can interrupt (continue) growth at any point in time. This allows us to perform accurate titration experiments on both the $5 \times 2$ and the $\alpha \sqrt{3} \times \sqrt{3}$ phases. In fact, a sample with a 


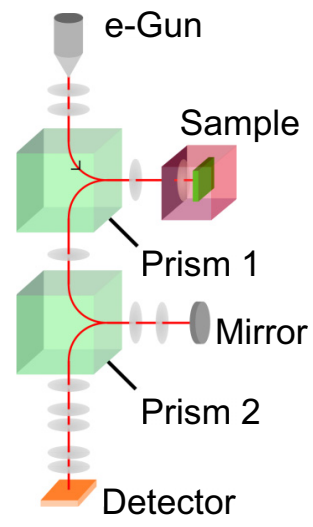

FIG. 1. (Color online) Schematic of the aberration corrected low energy electron microscope. The red trace shows the electron path from the gun via the sample and the mirror towards the detector, where an image is formed. The electrostatic mirror corrects for the aberrations of the objective lens. We can switch between real space (LEEM) and reciprocal space (LEED) imaging.

complete $5 \times 2$ or $\alpha \sqrt{3} \times \sqrt{3}$ structure can be taken out for ex-situ ion scattering experiments. Our dynamic experiments are performed in the so-called "Escher" setup, ${ }^{26}$ a state-ofthe-art aberration-corrected LEEM facility located at Leiden University. ${ }^{27-29}$ A schematic view of this UHV (ultrahigh vacuum) instrument is shown in Fig. 1. Electrons with an energy of $15 \mathrm{keV}$ are generated from the electron gun and deflected towards the objective lens and the sample by a double-focusing magnetic prism array. In the electric field between the objective lens and the sample (with the sample held at $\approx-15 \mathrm{kV}$ ) the electrons are decelerated to an energy of a few eV at the sample. The exact electron energy at the sample is tunable by the operator. At the sample the electrons undergo low energy electron diffraction, and the diffracted electrons (as well as secondary electrons) are accelerated back into the objective lens and directed again towards the magnetic prism array which deflects the electrons by $90^{\circ}$ towards a second magnetic prism array. After $90^{\circ}$ deflection in this second prism array, an electrostatic mirror corrects for the chromatic and spherical aberrations of the objective lens as it reflects the electrons back in a 1:1 image forming system. A final $90^{\circ}$ deflection in the second prism array directs the electrons towards the projector column and finally a channel plate detector. By changing the settings of the projector lenses, either the real space LEEM image or the LEED pattern can be observed in real time.

$\mathrm{Si}(111)$ substrates with a miscut of $0.1^{\circ}$ were annealed at $1250 \mathrm{~K}$ for 1 hour and then flash heated several times to $1450 \mathrm{~K}$ for 10 seconds, until a clean $7 \times 7$ reconstructed surface was obtained. Sample heating was done by electron bombardment from a tungsten filament behind the sample, while the temperature was monitored by an IMPAC IN 140 pyrometer. During this process the pressure never rose above $3 \times 10^{-9}$ mbar. After sample cleaning, each experiment started with in-situ gold evaporation onto the substrate from a home-built Knudsen cell evaporator. The evaporator consists of a boron nitride crucible with a tungsten filament coiled around it. The temperature of the crucible was measured using an S-type thermocouple and controlled with a Red Lion PID controller. A shutter, positioned between the Knudsen cell and the sample, was used to start or stop evaporation onto the sample at will.

To follow gold growth in detail, we use various imaging modes. Information on the surface structure is obtained by projecting the low-energy electron diffraction (LEED) pattern onto the detector. By changing the projector lens settings real-space LEEM images are obtained. To optimize contrast in the real-space images, we made use of both bright-field and dark-field imaging conditions. In bright-field LEEM an aperture is placed to select only the zeroth order LEED spot. We thereby utilize contrast between structures with different specular reflection intensities at certain energies (structure factor contrast). In dark-field LEEM, instead of the center spot, one of the other diffraction spots is selected. Then, the real-space image shows bright areas for those parts of the sample that contribute to the selected diffraction spot. For example, upon selecting one of the $5 \times 2$ spots, only those $5 \times 2$ domains that diffract into that spot become bright, while the rest of the surface (including the $5 \times 2$ domains that diffract to other LEED spots) remains dark in the image. We can then use the information obtained in dark field to interpret energy-dependent contrast in the bright-field images. Thus we determine that in bright-field images taken at an electron energy of $14.5 \mathrm{eV}$, the darker areas are $7 \times 7$ reconstructed and the brighter areas have a $5 \times 2$ structure. Similarly, at an electron energy of $8.2 \mathrm{eV}$ the $5 \times 2$ structure appears dark and the $\alpha \sqrt{3} \times \sqrt{3}$ structure appears bright.

\section{RESULTS}

Figure 2(a) shows individual LEEM video frames of the various stages of gold growth on $\mathrm{Si}(111)$, imaged in brightfield LEEM mode. For the first two images an electron energy of $14.5 \mathrm{eV}$ was used to obtain optimal contrast between the $7 \times 7$ (dark) and $5 \times 2$ (bright) reconstructions. As gold evaporation proceeds, the $7 \times 7$ areas reduce in size, until the $5 \times 2$ structure covers the entire surface at a time $t_{1}$. Shortly after, at $t=t_{1}^{\prime}$, the formation of $\alpha \sqrt{3} \times \sqrt{3}$ domains is observed. To follow their growth the electron energy is changed to $8.2 \mathrm{eV}$, thus maximizing contrast between the $5 \times 2$ (dark at this energy) and $\alpha \sqrt{3} \times \sqrt{3}$ (bright) phases. The third and fourth video frames in Fig. 2(a) show the growth of the $\alpha \sqrt{3} \times \sqrt{3}$ structure at the cost of the $5 \times 2$ phase. At a time $t_{2}$, the $\alpha \sqrt{3} \times \sqrt{3}$ phase reaches full coverage. To analyze the entire process in detail, we determine the surface fraction of the $7 \times 7,5 \times 2$, and $\alpha \sqrt{3} \times \sqrt{3}$ phases as a function of time. Figure 2(b) shows the results for the experiment in Fig. 2(a, with the relevant time scales $t_{1}, t_{1}^{\prime}$, and $t_{2}$ indicated. Additionally, Fig. 2(c) displays the LEED patterns observed at full coverage of the $5 \times 2$ and $\alpha \sqrt{3} \times \sqrt{3}$ structures, respectively (i.e., after stopping the process at $t_{1}$ and $t_{2}$ ). The $5 \times 2$ LEED pattern consists of three overlapping single domain $5 \times 2$ patterns rotated by $120^{\circ}$. The $5 \times$ spots are sharp, while the $2 \times$ periodicity appears as elongated streaks. The reason for these streaks is thought to lie in the observation that within one domain there are two ways to form a $5 \times 2$ unit cell. ${ }^{19,30,31}$ Mixing of these $5 \times 2$ unit cells leads to reduced order and a streaky LEED pattern. We used the $\sqrt{3} \times \sqrt{3}$ 
(a)

(b)
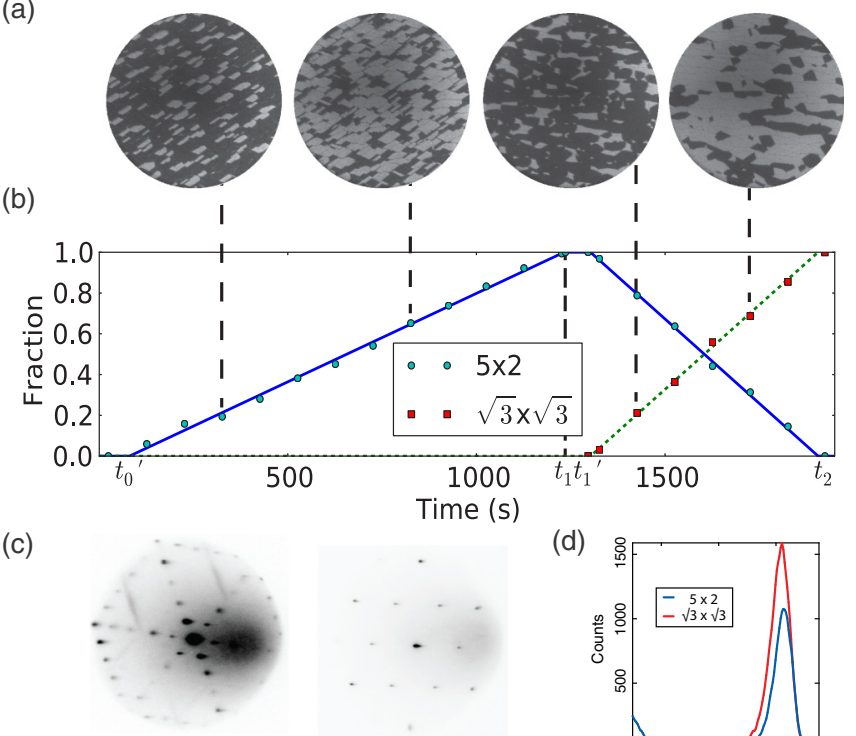

$5 \times 2$

$\sqrt{3} \times \sqrt{3}$

FIG. 2. (Color online) (a) LEEM video frames from an evaporation experiment at $920 \mathrm{~K}$ in bright-field imaging mode. Upon gold evaporation onto a bare $7 \times 7 \mathrm{Si}(111)$ sample, islands with the $5 \times 2$ structure are formed (bright in the first two frames). After the $5 \times 2$ islands cover the entire sample at time t1, we change the electron energy from 14.5 to $8.2 \mathrm{eV}$ to optimize contrast between $5 \times 2$ (dark) and $\alpha \sqrt{3} \times \sqrt{3}$ islands (bright). (b) Surface coverages of the $5 \times 2$ and $\alpha \sqrt{3} \times \sqrt{3}$ reconstructions versus time during gold evaporation. The fitted blue line shows the growth and decrease of the $5 \times 2$ coverage while the dashed green line gives the coverage of the $\alpha \sqrt{3} \times \sqrt{3}$ reconstructed area. Evaporation starts at $t=0$. The first $5 \times 2(\alpha \sqrt{3} \times \sqrt{3})$ islands become visible at $t_{0}^{\prime}\left(t_{1}^{\prime}\right)$ and completely fill the surface at $t_{1}\left(t_{2}\right)$. (c) LEED pattern of the $5 \times 2$ and $\alpha \sqrt{3} \times \sqrt{3}$ reconstructions taken at time $t_{1}$ and $t_{2}$, respectively. The dark shadows in both images are caused by secondary electrons. (d) MEIS results depicting the number of counts as a function of scattering energy for two samples with a complete $5 \times 2$ and $\alpha \sqrt{3} \times \sqrt{3}$ coverage, respectively, grown at $920 \mathrm{~K}$. By comparing the integrated area under the gold reflection peaks, we obtain the ratio between the two gold coverages. We find $\Theta_{5 \times 2} / \Theta_{\alpha \sqrt{3} \times \sqrt{3}}=0.65 \pm 0.01$.

LEED pattern to determine the size of the $\alpha \sqrt{3} \times \sqrt{3}$ domains. From the width of the $\sqrt{3} \times \sqrt{3}$ LEED spots we determined the average domain area to be large ( $\sim 13000$ unit cells) compared to the area of the domain boundaries. We can therefore ignore the influence of the domain boundaries on the coverage. ${ }^{32}$

Inspecting Fig. 2(b), we see that both the $5 \times 2$ and $\alpha \sqrt{3} \times \sqrt{3}$ phases grow linearly in time, but with different rates. We repeated this experiment for a range of growth temperatures from 820 to $970 \mathrm{~K}$, and consistently observed linear growth. [Scatter is generally due to the finite field of view and the resulting statistical limitations, see Fig. 2(a).] This linearity is important. Not only does it imply that the flux of gold coming onto the sample is constant, but also that there is no significant desorption, nor diffusion into the bulk at the time scale of this experiment. Indeed, since the latter processes depend on the gold coverage of the surface, they would lead to nonlinear growth. This conclusion is confirmed by the observation that the relative $5 \times 2$ and/or $\alpha \sqrt{3} \times \sqrt{3}$ coverages do not change with time after closing the evaporation shutter.

Since growth is linear the ratio between the gold coverages of the $5 \times 2$ and $\alpha \sqrt{3} \times \sqrt{3}$ reconstructions can be directly determined from $t_{1}$ and $t_{2}$, via $\Theta_{5 \times 2} / \Theta_{\alpha \sqrt{3} \times \sqrt{3}}=t_{1} / t_{2} .{ }^{4}$ Here, $\Theta_{5 \times 2}\left(\Theta_{\alpha \sqrt{3} \times \sqrt{3}}\right)$ denotes the gold coverage of the onset of the single-phase $5 \times 2(\alpha \sqrt{3} \times \sqrt{3})$ structure at a temperature T. From all growth experiments performed (eight in total with $820<T<970 \mathrm{~K}$ ), we determine an average value $\Theta_{5 \times 2} / \Theta_{\alpha \sqrt{3} \times \sqrt{3}}=0.65 \pm 0.02$. This result is fully consistent with the value obtained by Swiech et al. in a previous LEEM experiment, i.e., $\Theta_{5 \times 2} / \Theta_{\alpha \sqrt{3} \times \sqrt{3}}=$ $0.665 \pm 0.010 .{ }^{4}$ When we take for $\Theta_{\alpha \sqrt{3} \times \sqrt{3}}$ the commonly accepted value of $1.00,{ }^{16,24,25,33-35}$ our data lead to the remarkable conclusion that $\Theta_{5 \times 2}$ is significantly higher than 0.6 , the value on which the latest structural models are based.

To independently test this result, we combined accurate gold titration in LEEM with ex-situ ion scattering experiments, i.e., MEIS and RBS. We first evaporated gold onto a sample at $920 \mathrm{~K}$ and stopped deposition just before the sample was completely covered with a $5 \times 2$ phase. We then repeatedly opened the shutter for one second until the surface was just completely covered by the $5 \times 2$ structure. The gold coverage of the sample was then measured ex situ in a MEIS experiment. We repeated this experiment for a sample completely covered in an $\alpha \sqrt{3} \times \sqrt{3}$ phase. Two MEIS spectra, for $5 \times 2$ and $\alpha \sqrt{3} \times \sqrt{3}$, are shown in Fig. 2(d). To compare these data sets to the growth experiments, we consider the ratio between the two coverages. This automatically eliminates any systematic errors in the absolute calibration of MEIS. The value of $\Theta_{5 \times 2} / \Theta_{\alpha \sqrt{3} \times \sqrt{3}}$ is found to be $0.65 \pm 0.01$, matching the ratio determined from $t_{1}$ and $t_{2}$ in the LEEM growth experiments for the temperature range $820<T<970 \mathrm{~K}\left(\Theta_{5 \times 2} / \Theta_{\alpha \sqrt{3} \times \sqrt{3}}=\right.$ $0.65 \pm 0.02) .{ }^{36}$ As a final check we measured the gold coverage of the $\alpha \sqrt{3} \times \sqrt{3}$ sample using RBS. We found a coverage of $1.03 \pm 0.05$, which agrees with the commonly accepted value of 1.00 for the $\alpha \sqrt{3} \times \sqrt{3}$ structure, where one monolayer is defined as one atom per unreconstructed $\mathrm{Si}(111)$ surface unit cell. This demonstrates the consistency between the two types of experiments. We come to the conclusion that our set of experimental values for $\Theta_{5 \times 2}$ is significantly higher than the value of 0.6 which was used by Erwin and Abukawa. We will elaborate on this below.

Based on the data from these experiments we can start to form an experimental phase diagram of submonolayers of $\mathrm{Au}$ on $\mathrm{Si}(111)$. Our results can be critically compared to the work by Grozea et al. who proposed the most recent phase diagram by putting together results from several experimental studies. ${ }^{10}$ Figure 3(a) shows our data set. The cyan diamonds represent the absolute values obtained from the ion scattering experiments. The coverages obtained from the growth experiments are represented by red squares. At very low coverages, there is the boundary between the pure $7 \times 7$ reconstruction and the mixed phase region of $7 \times 7$ and $5 \times 2$ structures. The $5 \times 2$ phase is complete at $\Theta_{5 \times 2}(T) \approx 0.65$, with a slight variation with temperature. For somewhat higher coverages, denoted by the open red squares (defining $\Theta_{5 \times 2}^{\prime}$, determined from $t_{1}^{\prime} / t_{2}$ ), we observe domains of the $\alpha \sqrt{3} \times \sqrt{3}$ 


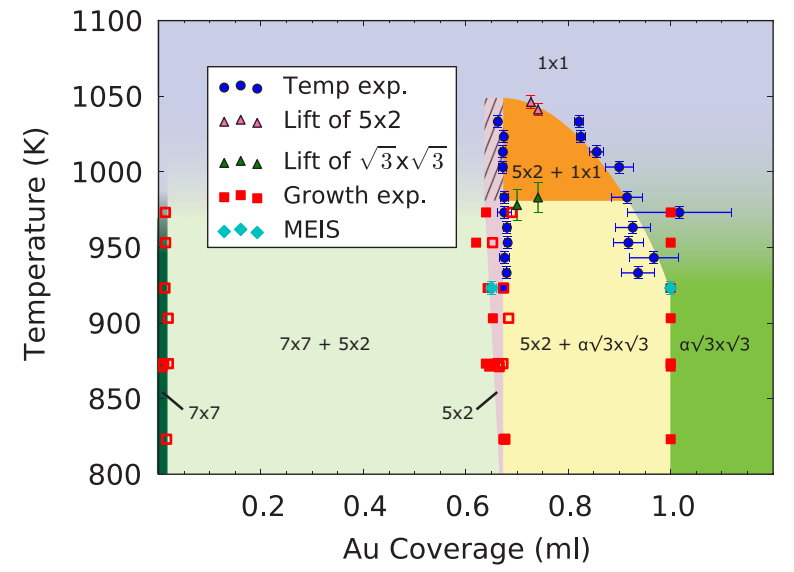

FIG. 3. (Color online) Experimental phase diagram for submonolayers of gold on $\mathrm{Si}(111)$. The cyan diamonds represent the MEIS results. The red squares are deduced from the times obtained in the evaporation experiments. The filled squares indicate the completion of the $5 \times 2$ and $\alpha \sqrt{3} \times \sqrt{3}$ phase, corresponding to $t_{1}$ and $t_{2}$, respectively. The open squares represent the first observation of domains of the $5 \times 2$ and $\alpha \sqrt{3} \times \sqrt{3}$ phase, corresponding to $t_{0}^{\prime}$ and $t_{1}^{\prime}$. For both the MEIS experiment and the growth experiment the value for completion of the $\alpha \sqrt{3} \times \sqrt{3}$ structure was fixed to 1.00 . The blue circles are the results from the temperature variation experiments in Fig. 4. The green and pink triangles depict the transition of the $\alpha \sqrt{3} \times \sqrt{3}$ and $5 \times 2$ phase, respectively, into the $1 \times 1$ phase, for coverages between $\Theta_{5 \times 2}^{\prime}$ and $\Theta_{\alpha \sqrt{3} \times \sqrt{3}}$. For lower and higher coverages, the estimated melting temperatures have been depicted as smooth transitions in the phase diagram. ${ }^{10}$ Our results show that the pure $5 \times 2$ phase has a finite width that increases with temperature. Above $920 \mathrm{~K}$ we only know the high coverage boundary of the $5 \times 2$ coverage. The hatched area depicts how we expect the $5 \times 2$ phase to continue.

phase for the first time. Finally we reach the pure $\alpha \sqrt{3} \times \sqrt{3}$ phase at a coverage of $1.00 .{ }^{37}$

At higher temperatures $(T>970 \mathrm{~K})$ growth experiments could not be done with sufficient accuracy, mainly due to increasing domain sizes which become large compared to the field of view. To extend the phase diagram at these temperatures, and specifically to follow the behavior of the $5 \times 2$ structure, we performed a fourth type of experiment. For this, we again evaporated gold at $920 \mathrm{~K}$, but this time we stopped in the two-phase region $(5 \times 2$ and $\alpha \sqrt{3} \times \sqrt{3})$. We then repetitively heated and cooled the sample while observing the surface in dark field. After each temperature step, $T$ was kept constant for at least 20 seconds to allow the system to achieve a stable configuration. Then, we acquired four dark-field images, using a different diffraction spot for each image: One image was obtained using a $\alpha \sqrt{3} \times \sqrt{3}$ diffraction spot, and three images were obtained using one of the $2 / 5$ order diffraction spots for each of the three $5 \times 2$ LEED pattern orientations. Subsequently, the temperature was changed again, etc. The full experiment thus allowed us to follow the evolution of the individual orientations of the $5 \times 2$ structure (and the total $5 \times 2$ area) as well as the $\alpha \sqrt{3} \times \sqrt{3}$ domains (and their total area) as a function of temperature. In total, we performed three such sequences, each time starting with a different Au coverage.

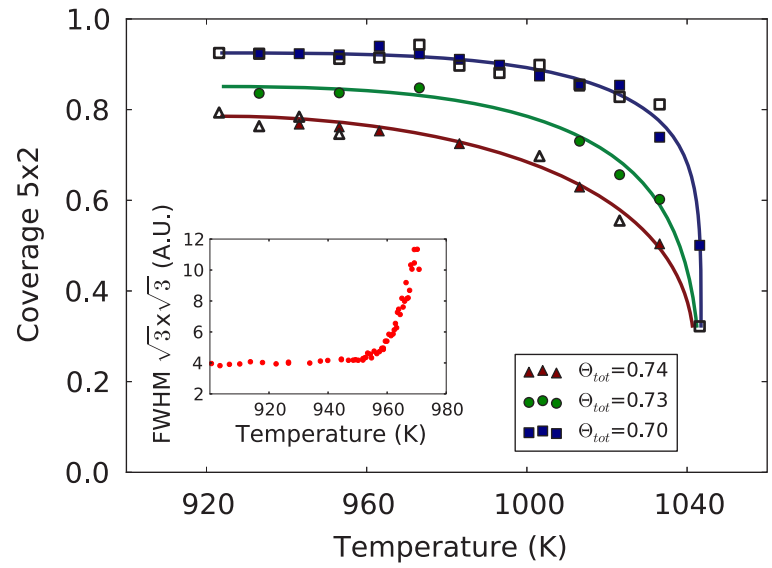

FIG. 4. (Color online) Temperature dependence of the $5 \times 2$ coverage for three samples with different starting coverages for the $5 \times 2$ and $\alpha \sqrt{3} \times \sqrt{3}$ reconstructions. Filled and open markers correspond to measurements after respectively raising and lowering the temperature. The lines are guides to the eye. As we increase the temperature the $5 \times 2$ coverage reduces. Since the total amount of gold on the sample remains constant, the number of gold atoms in the $5 \times 2$ and $\alpha \sqrt{3} \times \sqrt{3}$ reconstruction, respectively, must be changing. Inset: FWHM of the $\sqrt{3} \times \sqrt{3}$ peak as a function of temperature. Around $980 \mathrm{~K}$ the $\alpha \sqrt{3} \times \sqrt{3}$ structure melts into a disordered $1 \times 1$ phase.

We observe two systematic changes with increasing temperature: (i) the $\sqrt{3} \times \sqrt{3}$ dark-field images gradually lose contrast; (ii) the $5 \times 2$ domains reduce in size and coverage. The first observation can be attributed to a transition from the $\alpha \sqrt{3} \times \sqrt{3}$ phase to the disordered $1 \times 1$ phase. During this transition the surface gets increasingly disordered, leading to a broadening of the $\sqrt{3} \times \sqrt{3}$ diffraction spots. The evolution of the full width at half maximum (FWHM) of the $\alpha \sqrt{3} \times \sqrt{3}$ diffraction spots with increasing temperature is depicted in the inset in Fig. 4. At $980 \mathrm{~K}$ there is a clear phase transition from the $\alpha \sqrt{3} \times \sqrt{3}$ phase to the $1 \times 1$ phase. The transition is rather sharp and takes place within approximately $20 \mathrm{~K}$. This phase transition is depicted in Fig. 3 by the green triangles. The second observation is a reduction of the total $5 \times 2$ area upon heating. In Fig. 4 we plot the temperature dependence of the total coverage of the $5 \times 2$ domains, normalized to the full field of view. Importantly, we find no significant difference between values obtained after coming from a lower temperature and those obtained after coming from a higher temperature. This reversibility of the transitions between $5 \times 2$ and $\alpha \sqrt{3} \times \sqrt{3}$ again indicates that there is no significant desorption or bulk diffusion of gold on the timescale of the experiment. Hence, the total amount of gold on the sample remains constant and we can use the following relation to connect Fig. 4 to the phase diagram in Fig. 3:

$$
f_{5 \times 2}(T) \Theta_{5 \times 2}^{\prime}(T)+\left(1-f_{5 \times 2}(T)\right) \Theta_{\alpha \sqrt{3} \times \sqrt{3} / 1 \times 1}(T)=\Theta_{\text {tot }},
$$

where $f_{5 \times 2}$ denotes which fraction of the sample is covered by $5 \times 2$ domains, $\Theta_{5 \times 2}^{\prime}$ represents the gold coverage of the $5 \times 2$ phase at the border with the mixed phase region, and $\Theta_{\alpha \sqrt{3} \times \sqrt{3} / 1 \times 1}$ is the gold content of the non-5 $\times 2$ areas, which 
are either in the $\alpha \sqrt{3} \times \sqrt{3}$ or $1 \times 1$ phase, depending on the temperature. The total amount of gold on the sample, $\Theta_{\text {tot }}$, is calculated by filling in the starting coverages at $920 \mathrm{~K}$, together with the values for $\Theta_{5 \times 2}^{\prime}$ and $\Theta_{\alpha \sqrt{3} \times \sqrt{3}}$. The fact that the total area occupied by $5 \times 2$ domains decreases with temperature must mean that $\Theta_{5 \times 2}^{\prime}(T)$ and/or $\Theta_{\alpha \sqrt{3} \times \sqrt{3} / 1 \times 1}(T)$ change with temperature. Combining the information from the three different experiments in Fig. 4, we can fit values for $\Theta_{5 \times 2}^{\prime}(T)$ and $\Theta_{\alpha \sqrt{3} \times \sqrt{3} / 1 \times 1}(T)$ for each temperature. The results are depicted in Fig. 3, further completing our phase diagram. We note that for our experiments we have deliberately chosen three gold concentration values, $\Theta_{\mathrm{tot}}$, close to the coverage of the pure $5 \times 2$ structure. Hence, we are very sensitive to changes in the coverage of the $5 \times 2$ structure. We can therefore conclude that $\Theta_{5 \times 2}^{\prime}$ remains essentially constant at the temperatures chosen, with an average value of $0.67 \pm 0.01$. We have thus completed our experimental determination of all the boundaries of the mixed $5 \times 2+\alpha \sqrt{3} \times \sqrt{3}$ phase. Figure 3(a) shows the resulting phase diagram. Both the $7 \times 7+\mathrm{Au}$ and $5 \times 2$ phases are found to have a finite width, i.e., the structures can incorporate adatoms or vacancies. We stress that both the left and right boundaries of the $5 \times 2$ phase, $\Theta_{5 \times 2}=0.65$ and $\Theta_{5 \times 2}^{\prime}=0.67$, respectively, are significantly higher than the value of 0.6 used in the latest structural models.

\section{DISCUSSION}

We have performed four types of experiments and thereby accurately determined the boundaries of the phase diagram. When we compare our results to the previous phase diagram by Grozea, ${ }^{10}$ one immediately notices a difference in scaling due to the assumption of a lower $\alpha \sqrt{3} \times \sqrt{3}$ coverage already mentioned before. A more profound difference between our phase diagram and that of Grozea is visible at the top of the $\alpha \sqrt{3} \times \sqrt{3}$ phase. In the phase diagram from Grozea, a peritectic point is drawn at the top of the $\alpha \sqrt{3} \times \sqrt{3}$ phase, i.e., when a $\alpha \sqrt{3} \times \sqrt{3}$ phase is heated until above its melting temperature, a mixture of $5 \times 2$ and $1 \times 1$ domains will form. In our heating experiments such a phase transition would lead to an increase in $5 \times 2$ coverage when passing this temperature during heating. No such behavior was observed during our experiments. We therefore conclude that this peritectic point is either not there, or its position is very near to the boundary with the $1 \times 1$ phase.

Our most important result is that we find a coverage for the $5 \times 2$ phase between $0.65 \pm 0.02$ and $0.67 \pm 0.01$, which does not agree with the latest structural models by Erwin and Abukawa, who assume a gold coverage of 0.6 monolayer. However, our findings are within experimental errors in good agreement with earlier experimental results. ${ }^{4,12,16}$ A simple solution to the discrepancy between our findings and the models would be that the adatoms which are part of the $5 \times 2$ reconstruction would be gold atoms instead of silicon atoms. This would lead to a gold coverage for the $5 \times 2$ phase of 0.65 . This option has been discussed intensively over the years, $, 38,39$ but was deemed less probable after experiments by Baski ${ }^{40}$ and later by Kirakosian. ${ }^{31}$ Whether the explanation is as simple as changing the type of adatoms, or that the model has to be altered more fundamentally, it is clear that the structural model for the $5 \times 2$ reconstruction has to be reconsidered.

\section{CONCLUSIONS}

We have analyzed (sub)monolayers of gold on $\mathrm{Si}(111)$ using LEEM, LEED, and MEIS. From our results we were able to determine the locations of the phase boundaries in the $\mathrm{Au} / \mathrm{Si}(111)$ phase diagrams to form a phase diagram for submonolayers of gold on $\mathrm{Si}(111)$. Our results show a gold content for the $5 \times 2$ structure between $0.65 \pm 0.02$ and $0.67 \pm 0.01$ monolayers, thereby creating the need for an improved structural model.

\section{ACKNOWLEDGMENTS}

We are grateful to Marcel Hesselberth and Daan Boltje for technical assistance and to Joost Frenken, Frank Meyer zu Heringdorf, and Geert Brocks for useful discussions. This work was supported by the Netherlands foundation for scientific research (NWO) by means of a "NWO Groot" and a "VIDI" grant and by the FOM foundation via the "Physics in 1D" program.

\footnotetext{
*Corresponding author: molen@physics.leidenuniv.nl

${ }^{1}$ G. Le Lay, Surf. Sci. 132, 169 (1983).

${ }^{2}$ K. Oura, M. Katayama, F. Shoji, and T. Hanawa, Phys. Rev. Lett. 55, 1486 (1985).

${ }^{3}$ E. Bauer, Surf. Sci. 250, L379 (1991).

${ }^{4}$ W. Swiech, E. Bauer, and M. Mundschau, Surf. Sci. 253, 283 (1991).

${ }^{5}$ C. Schamper, W. Moritz, H. Schulz, R. Feidenhans'1, M. Nielsen,

F. Grey, and R. L. Johnson, Phys. Rev. B 43, 12130 (1991).

${ }^{6}$ J. Yuhara, M. Inoue, and K. Morita, J. Vac. Sci. Technol., A 10, 334 (1992).

${ }^{7}$ J. D. O’Mahony, J. F. McGilp, C. F. J. Flipse, P. Weightman, and F. M. Leibsle, Phys. Rev. B 49, 2527 (1994).

${ }^{8}$ L. D. Marks and R. Plass, Phys. Rev. Lett. 75, 2172 (1995).

${ }^{9}$ R. Plass and L. D. Marks, Surf. Sci. 380, 497 (1997).

${ }^{10}$ D. Grozea, E. Bengu, and L. D. Marks, Surf. Sci. 461, 23 (2000).
}

${ }^{11}$ K. N. Altmann, J. N. Crain, A. Kirakosian, J.-L. Lin, D. Y. Petrovykh, F. J. Himpsel, and R. Losio, Phys. Rev. B 64, 035406 (2001).

${ }^{12}$ R. Hild, C. Seifert, M. Kammler, F.-J. Meyer zu Heringdorf, M. Horn-von Hoegen, R. A. Zhachuk, and B. Z. Olshanetsky, Surf. Sci. 512, 117 (2002).

${ }^{13}$ S. C. Erwin, Phys. Rev. Lett. 91, 206101 (2003).

${ }^{14}$ J. N. Crain, J. L. McChesney, F. Zheng, M. C. Gallagher, P. C. Snijders, M. Bissen, C. Gundelach, S. C. Erwin, and F. J. Himpsel, Phys. Rev. B 69, 125401 (2004).

${ }^{15}$ S. Riikonen and D. Sánchez-Portal, Phys. Rev. B 71, 235423 (2005).

${ }^{16}$ I. Barke, F. Zheng, S. Bockenhauer, K. Sell, V. Oeynhausen, K. H. Meiwes-Broer, S. C. Erwin, and F. J. Himpsel, Phys. Rev. B 79, 155301 (2009).

${ }^{17}$ S. C. Erwin, I. Barke, and F. J. Himpsel, Phys. Rev. B 80, 155409 (2009). 
${ }^{18}$ N. McAlinden and J. F. McGilp, Europhys. Lett. 92, 67008 (2010).

${ }^{19}$ T. Abukawa and Y. Nishigaya, Phys. Rev. Lett. 110, 036102 (2013).

${ }^{20}$ The current structural models do not exhibit mirror symmetry in the $2 \times$ direction, hence there are actually six possible orientations. Experimentally we cannot distinguish two orientations rotated by $180^{\circ}$.

${ }^{21}$ T. Hasegawa, S. Hosaka, and S. Hosoki, Surf. Sci. 357-358, 858 (1996).

${ }^{22}$ C. Hogan, E. Ferraro, N. McAlinden, and J. F. McGilp, Phys. Rev. Lett. 111, 087401 (2013).

${ }^{23}$ W. Telieps and E. Bauer, Ultramicroscopy 17, 57 (1985).

${ }^{24}$ M. Chester and T. Gustafsson, Surf. Sci. 256, 135 (1991).

${ }^{25}$ T. Nagao, S. Hasegawa, K. Tsuchie, S. Ino, C. Voges, G. Klos, H. Pfnür, and M. Henzler, Phys. Rev. B 57, 10100 (1998).

${ }^{26}$ S. M. Schramm, J. Kautz, A. Berghaus, O. Schaff, R. M. Tromp, and S. J. van der Molen, IBM J. Res. Dev. 55, 1 (2011).

${ }^{27}$ R. M. Tromp, J. B. Hannon, A. W. Ellis, W. Wan, A. Berghaus, and O. Schaff, Ultramicroscopy 110, 852 (2010).

${ }^{28}$ R. M. Tromp, J. B. Hannon, W. Wan, A. Berghaus, and O. Schaff, Ultramicroscopy 127, 25 (2013).

${ }^{29}$ S. M. Schramm, A. B. Pang, M. S. Altman, and R. M. Tromp, Ultramicroscopy 115, 88 (2012).

${ }^{30}$ J. D. O’Mahony, C. H. Patterson, J. F. McGilp, F. M. Leibsle, P. Weightman, and C. F. J. Flipse, Surf. Sci. 277, L57 (1992).

${ }^{31}$ A. Kirakosian, R. Bennewitz, F. J. Himpsel, and L. W. Bruch, Phys. Rev. B 67, 205412 (2003).
${ }^{32}$ The width of the LEED spots puts an upper bound on the increase in gold coverage induced by the domain boundaries. If we assume a domain wall width of two unit cells and a gold coverage of the domain boundaries of $\Theta_{\text {domainboundary }}=1.73,{ }^{25,33}$ the maximum increase in gold coverage is $\sim 2 \%$.

${ }^{33}$ D. V. Gruznev, I. N. Filippov, D. A. Olyanich, D. N. Chubenko, I. A. Kuyanov, A. A. Saranin, A. V. Zotov, and V. G. Lifshits, Phys. Rev. B 73, 115335 (2006).

${ }^{34}$ C.-H. Hsu, W.-H. Lin, V. Ozolins, and F.-C. Chuang, Phys. Rev. B 85, 155401 (2012).

${ }^{35}$ In the paper by Swiech et al. ${ }^{4} \Theta_{\alpha \sqrt{3} x \sqrt{3}}=2 / 3$ was assumed, leading to the low values mentioned in the introduction: $\Theta_{5 \times 2} \approx 0.4$.

${ }^{36}$ For the particular temperature at which the MEIS samples were grown $(920 \mathrm{~K})$, the growth experiments yield $\Theta_{5 \times 2} / \Theta_{\alpha \sqrt{3} \times \sqrt{3}}=$ $0.64 \pm 0.02$.

${ }^{37}$ Note that we fixed the values for the completed $\alpha \sqrt{3} \times \sqrt{3}$ structure in the growth experiments, $\Theta_{\alpha \sqrt{3} \times \sqrt{3}}$, to the gold coverage obtained for the $\alpha \sqrt{3} \times \sqrt{3}$ phase (1.00). This automatically determines the other values of the growth experiments, specifically those for the $5 \times 2$ structure. Alternative choices, e.g., fixing $\Theta_{5 \times 2}$ to the MEIS value of 0.67 does not lead to significant changes in the phase diagram. The value for $\Theta_{5 \times 2}$ always remains around 0.65 .

${ }^{38}$ T. Hasegawa and S. Hosoki, Phys. Rev. B 54, 10300 (1996).

${ }^{39}$ I. G. Hill and A. B. McLean, Phys. Rev. B 55, 15664 (1997).

${ }^{40}$ A. A. Baski, J. Nogami, and C. F. Quate, Phys. Rev. B 41, 10247 (1990). 